\title{
A Study on Pre-processing Algorithms for Metal Parts Inspection
}

\author{
Haider Sh. Hashim, Anton Satria Prabuwono, Siti Norul Huda Sheikh Abdullah \\ Center for Artificial Intelligence Technology (CAIT), \\ Faculty of Information Science and Technology, \\ University Kebangsaan Malaysia, 43600 UKM Bangi, Selangor D.E., Malaysia \\ haider_hashim76@yahoo.com; antonsatria,mimi\{@ftsm.ukm.my\}
}

\begin{abstract}
Pre-processing is very useful in a variety of situations since it helps to suppress information that is not related to the exact image processing or analysis task. Mathematical morphology is used for analysis, understanding and image processing. It is an influential method in the geometric morphological analysis and image understanding. It has befallen a new theory in the digital image processing domain. Edges detection and noise reduction are a crucial and very important pre-processing step. The classical edge detection methods and filtering are less accurate in detecting complex edge and filtering various types of noise. This paper proposed some useful mathematic morphological techniques to detect edge and to filter noise in metal parts image. The experimental result showed that the proposed algorithm helps to increase accuracy of metal parts inspection system.
\end{abstract}

\section{INTRODUCTION}

Pre-processing is very useful in a variety of situations since it helps to suppress information that is not related to the exact image processing or analysis task. Image filtering and edge detection is an indispensable and necessary operation in image pre-processing step [1]. When the image is acquired, the factors such as the projection, mix, aberrance and noise are produced. These factors can lead to image blurring and distortion; consequently it can cause difficulty in extracting important feature in the image [2] like edges of objects in image. Mathematical morphology is a mathematical method using structural elements to analyze image, which has been widely applied to variety fields [3] like visual inspection. The morphological operations work to simplify image and preserve the main shape characteristics of objects. Morphological operators are mainly used in image preprocessing for (noise filter, shape simplification and enhancing object structure) [4]. It provides an option approach to image processing depending on a shape set theory [5]. Mathematical morphology is a powerful tool to filter different types of noise and to detect the complex edge in variety directions $[1,2]$. Edge detection is one of the basal contents in the image processing and analysis, which is unable to be resolved completely so far [6]. This paper objective is to evaluate pre-processing algorithms for metal parts inspection system. We organize this paper into five sections. The second and third sections describe the basic mathematical morphology theory and proposed methodology subsequently. Then, the forth section explains the experimental evaluations. Finally, the last section wraps up the paper.

\section{BASIC MATHEMATICAL MORPHOLOGY THEORY}

Morphology operators like dilation and erosion with structuring elements (SE) are the most fundamental operators in mathematical morphology and have become common tools for both image filtering and analysis [3]. In 1960s, Matheron introduced mathematical morphology as a method for analyzing a geometric structure of metallic and geologic samples. Later, Serra is developed the method for image analysis. Mathematical morphology is a very significant theory, whose operation must be defined by using arithmetic sets [2]. Consequently, the resultant image posses two sets of different criteria such as determining either black and white pixel at specific location $(x, y)$ based on mathematical morphology like addition, multiplication or etc. It is an influential tool for dealing with a variety of problems in image processing and machine vision [3]. Basically, mathematical morphology consists of a series of morphological algebraic arithmetic operators. The essential morphological operations are dilation, erosion, closing, opening. Those operators are used for detecting, modifying, manipulating features in a predetermined image depending on their shapes. In line with that, morphological operators are closely related to the size and shape of structuring element (SE). SE depends on the purpose and requirements of application connected to the image [4]. We preview the basic of mathematical morphological operators of gray-scale images. Suppose $f(x, y)$ is an input image, $b(x, y)$ and $D f$ is a $\mathrm{SE}$, and $\mathrm{Db}$ are the domains of the input image and the SE, respectively:

Definition 1: Dilation operation $(f \oplus b)$, defined as, $(f \oplus b)(s, t)=\max \{f((s-x, t-y)+b(x, y) / s-x, t-y) \in$ $D f,(x, y) \in D b\}$

Definition 2: Erosion operation $(f \ominus b)$, defined as, $(f \ominus b)(s, t)=\max \{f((s+x, t+y)-b(x, y) / s+x, t+y) \in$ $D f,(x, y) \in D b\}$

Definition 3: Opening operation $(f \odot b)$, defined as, $(f \odot b)=((f \ominus b) \oplus b)$ 
Definition 4: Closing operation $(f \odot b)$, defined as, $(f \odot b)=((f \oplus b) \ominus b)$

From the definition 1 and 2, erosion is a change of shrinking, which decreases the specific pixel detail in an image, while dilation is a change of expanding, which increases the specific pixel detail in an image. However, both methods are sensitive to the image edge whose value changes apparently. Erosion filters the inner boundary of region of interest (ROI) in an image while dilation filters the outer ROI image. From the definition 3 and 4, opening is erosion followed by dilation, while closing is dilation followed by erosion. The opening operation is employed for removing small bright details (peak noise) smaller than the structural elements, smoothing the boundary of a ROI image, breaking narrow gaps, whilst the closing operation is applied for eliminating the small dark details (low noise), smaller than the structural elements, fusing narrow breaks or gaps, eradicating small holes and filling up gaps in the contours of ROI image [1]. Consequently, combining these two operations for image filtering can effectively remove peak and reduce noise in an image. As conclusion, morphological operations are usually used to detect image edge and to remove unwanted information in the image.

\section{PROPOSED FRAMEWORK}

Fig. 1 shows the proposed framework for metal parts visual inspection. The pre-processing steps are necessary for object recognition, noise reduction and edge detection. An image enhancement is aimed to provide a better image compared to the source image. We remove image noise and detect edge of objects by using mathematical morphology with SE. In conjunction to metal parts inspection, a postprocessing algorithm evaluation is conducted to classify the right metal parts in real-time inspection.

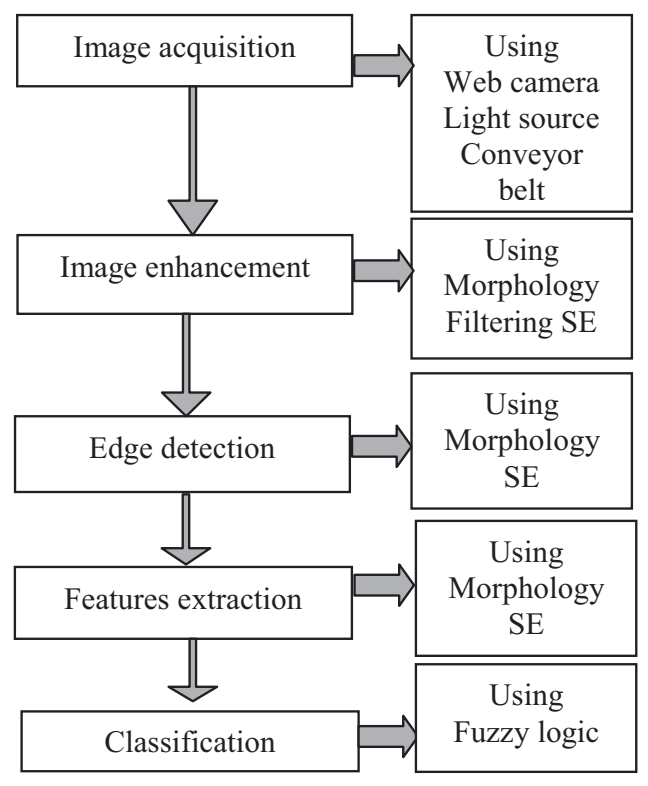

Figure 1. Proposed framework for metal parts visual inspection.

\section{A. Pre-processing}

Pre-processing is one of important steps in image processing. We apply some useful basic morphological operations such as opening and closing operators.

\section{1) Image Enhancement}

The morphological SE filter has a strong adaptability. It has good filtering effects on three different kinds of noise. Besides, it is powerful to filter out peak and low noise (salt and pepper). It can also maintain the detailed information of image. The most basic morphological operations are dilation and erosion. We apply some useful basic morphological operations such as Opening and Closing Operators.

\section{2) Edge Detection}

An edge is a sharply change of intensity in the image. The techniques can be classified based on first order derivative and second order derivative. The first technique uses gradient vector and estimates the gradient direction, e.g. Roberts, Sobel, and Prewitt. Then, the second technique uses the zerocrossing of the Laplacian or non-linear differential [7]. We apply the Morphological filter and edge detection based on SE. Generally, it is show a stronger method to filtering effect for images with peak noise (such as speckle noise), and it can work with effectively preserve the details of the images and detects the important edges.

Based on experimental results, we could only achieve satisfactory results for real images after using diamond SE with $5 \times 5$ window size as shown in Fig. 2(a). We choose $0^{\circ}, 15^{\circ}, 30^{\circ}, 45^{\circ}, 60^{\circ}, 75^{\circ}, 90^{\circ}, 105^{\circ}, 130^{\circ}, 145^{\circ}, 160^{\circ}$ for the direction angles as illustrated in Fig. 2(b) [8].

\begin{tabular}{|c|c|c|c|c|}
\hline$\bullet$ & $\bullet$ & 1 & $\bullet$ & $\bullet$ \\
\hline$\bullet$ & 1 & 1 & 1 & $\bullet$ \\
\hline 1 & 1 & 1 & 1 & 1 \\
\hline$\bullet$ & 1 & 1 & 1 & $\bullet$ \\
\hline$\bullet$ & $\bullet$ & 1 & $\bullet$ & $\bullet$ \\
\hline
\end{tabular}

Fig.2 (a). Simple of SE filters with $5 \times 5$.

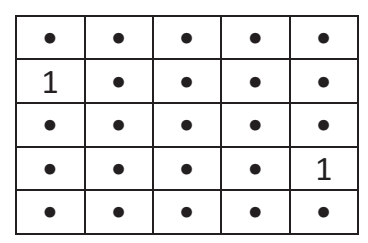

Fig.2 (b). Simple of SE edge detection with $5 \times 5$; angle of $145^{\circ}$.

\section{B. Post-processing}

In the post processing, we use fuzzy logic to classify the metal part. Fuzzy logic provides a natural method for constructing systems that emulate human decision making processes. Fuzzy ruled-based systems are knowledge based systems in which the knowledge is stored in a knowledge base that consists of three components: linguistic variables, membership functions, and a set of "IF-THEN" rules. Fuzzy inference systems have been successfully applied in fields such as automatic control, data classification, decision analysis, expert systems, and computer vision. There are mainly two types of fuzzy inference systems: 1) the Mamdani type and 2) the Sugeno type. The main difference between the Mamdani and Sugeno types is that the Sugeno output membership functions are either linear or constant. Based on our experimental results, we obtained an optimized model of this problem using Mamdani fuzzy inference rule [9]. 


\section{EXPERIMENTAL RESULT}

In this section, we present the experiment results. The proposed algorithm of edge detection and image filtering used mathematic morphological SE and compare with a different of traditional edge detection methods. Fig. 3(a) shows the original image that captured using web cam with size of $480 \times 640$. We tested on 10 images where the total number of actual objects is about 100 ROI $(10$ objects $\times 10$ images). We then classified those ROI using Mamdani fuzzy logic into 10 classes such as screw, big shim, small shim, medium shim, very small shim, slim bolt, short thin bolt, long thin bolt, big nut, and medium nut. To evaluate the overall performances, we calculate the accuracy as the followings:

$$
\text { The accuracy }=\frac{\text { total number of classified objects }}{\text { total number of actual objects }}
$$

Fig. 4(a) and Fig. 5(a) show an original image with salt and pepper noise. Furthermore, Fig. 3(b), 3(c), and 3(d) are the results after applying Gaussian filter, Sobel operator and after classifying using fuzzy rule respectively. Fig. 4(b), 4(c), and $4(d)$ are the results after applying Average filter, Canny operator and after classifying using fuzzy rule subsequently. Finally Fig. 5(b), 5(c), and 5(d) are the results using the proposed algorithm.

From Table I, Sobel with Gaussian filter, Canny with Average filter and proposed framework obtained about 55\%, $63 \%$ and $97 \%$ respectively. As conclusion, we can conclude that the proposed framework can detect edge and filter noise better than other conventional methods.

TABLE I. THE ACCURACY OF CLASSIFICATION

\begin{tabular}{|c|c|c|c|}
\hline & $\begin{array}{c}\text { Sobel } \\
\text { \& Gaussian }\end{array}$ & $\begin{array}{c}\text { Canny } \\
\text { \& Average }\end{array}$ & $\begin{array}{c}\text { Proposed } \\
\text { method }\end{array}$ \\
\hline Total no. of object & 100 & 100 & 100 \\
\hline Classified accuracy & $55 \%$ & $63 \%$ & $97 \%$ \\
\hline
\end{tabular}

\section{CONCLUSION}

In this paper, mathematical morphological operators are used for noise filtering whilst the edge detection using boundary tracing algorithm is proposed. The experimental result showed the proposed algorithm is more efficient than the conventional operator used such as Sobel with Gaussian and Canny with Average. The Morphological SE result is clear and continual edges. It provides all important information of object under inspection process. Sobel operator could be applied only for two directions e.g. vertical and horizontal. It use small mask to scan the whole image namely time consuming and did not detect the edge with any angle directions. Canny can detect a lot of information of image. It is not suitable to apply in machine vision since it needs to filter the information for the next process. The proposed algorithm could detect the edge in different and complex direction and show clear edge, pinpointed, integral and continual results. The final results showed the accuracy of proposed framework was $97 \%$.

\section{ACKNOWLEDGMENT}

Special thanks to Dr. Adnan Jabir Mr. Hassan Majeed who had worked hard in giving ideas and to effort in various areas. This project research is funded by Research University Operation No. OUP-ICT-36-186/2010, Junior Researcher Grant No. UKM-GGPM-ICT-103-2010 and UKM-AP-ICT17-2009.

\section{REFERENCES}

[1] Y. Zhao, W. Gui, and Z. Chen, "Edge detection based on multistructure elements morphology," Proc. the $6^{\text {th }}$ World Congrees on Intelligent Control and Automation, pp. 9795-9768, Dalian, October 2006.

[2] F.Y. Cui, L.J. Zou, and B. Song, “Edge feature extraction based on digital image processing techniques," Proc. IEEE International Conference on Automation and Logistics, pp. 2320-2324, Qingdao, 30 September 2008.

[3] G.B. Xu, Z.B. Su, J. Wang, Y.X. Yin, and Y.L. Shen, ”An adaptive morphological filter based on multiple structure and multi-scale elements," Proc. Second International Symposium on Intelligent Information Technology Application, Vol. 2, pp. 399-403, Shanghai, 6 January 2008.

[4] Y. Tingfang, L. Pei, Z. Xiangjun, and K.K. Li, "Application of adaptive generalized morphological filter in disturbance identification for power system signatures," Proc. International Conference on Power System Technology, pp. 1-7, Chongqing, 2006.

[5] Y.T. Hsiao, C.L. Chauang, J.A. Jiang, and C.C. Chien, "A contour based image segmentation algorithm using morphological edge detection," Proc. IEEE Internaional Conference on Systems Man and Cybernetics, Vol. 3, pp. 2962-2967, 2005.

[6] H. Akbar, and A.S. Prabuwono, "Webcam based system for press part industrial inspection," International Journal of Computer Science and Network Security, Vol. 8, No. 10, pp. 170-177, October 2008.

[7] M.I. Rajab, M.S. Woolfson, and S.P. Morgan, "Application of regionbased segmentation and neural network edge detection to skin lesions," Computerized Medical Imaging and Graphics, Vol. 28, No. 1-2, pp. 6168, January 2004.

[8] E.R. Urbach, and M.H.F. Wilkinson, "Efficient 2-D grayscale morphological transformations with arbitrary flat structuring elements," IEEE Transaction on Image Processing, Vol. 17, No. 1, pp.1-8, Jan 2008.

[9] H.Sh. Hashim, S.N.H.S. Abdullah, and A.S. Prabuwono, "Automated visual inspection for metal parts based on morphology and fuzzy rules," Proc. International Conference on Computer Application \& Industrial Electronics, Kuala Lumpur, 2010. 


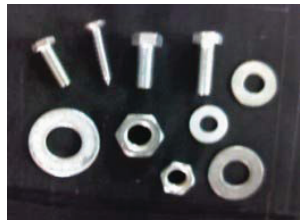

(a)

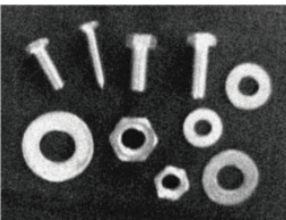

(b)

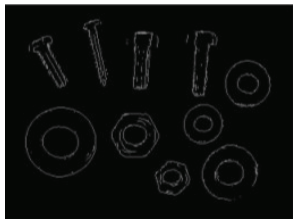

(c)

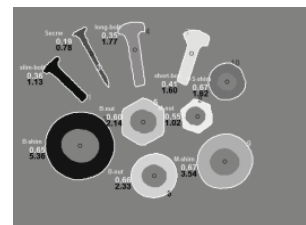

(d)

Figure 3. The result of experiment using Gaussian and Sobel: (a) Original Image size $480 \times 640$, (b) Image filtered by Gaussian filter, (c) The results of edge detection using Sobel operator, (d) Classification uses Fuzzy Rules.

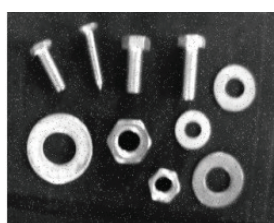

(a)

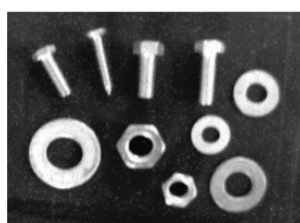

(b)

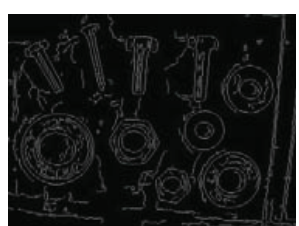

(c)

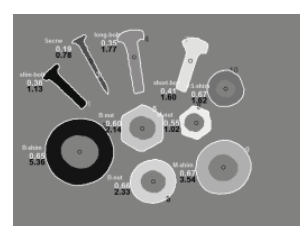

(d)

Figure 4. The result of experiment using Average and Canny: (a) Image with Salt and Pepper noise, (b) Image filtered by Average filter, (c) The results of edge detection used Canny operator, (d) Classification uses Fuzzy Rules.

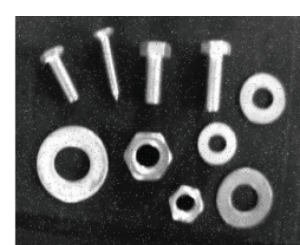

(a)

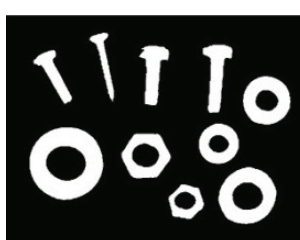

(b)

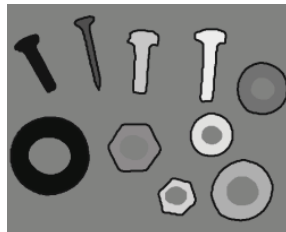

(c)

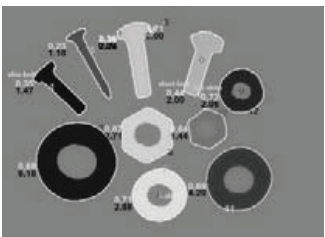

(d)

Figure 5. The result of experiment using proposed algorithm: (a) Image with Salt and Pepper noise, (b) Image filtered by Structure elements filter, (c ) The results of edge detection using structure elements morphology, (d ) Classification uses Fuzzy Rules. 